\title{
Does SAE have /r/? Evidence from Canadian Raising and Vowel Durations
}

\author{
Bethany Dickerson \\ Michigan State University, currently at University of Maryland
}

\section{Introduction}

When morphophonemic alternations do not provide evidence about the underlying phonological representation of an ambiguous segment, language users are faced with a situation in which there exist several viable underlying representations. For example, the [r] in Ryder [InIr $x]$ does not alternate with the allophones

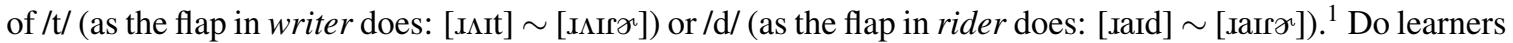
posit a faithful underlying $/ \mathrm{r} /$ (as in /.air $2 /$ ) or map the non-alternating $[\mathrm{r}]$ onto $/ \mathrm{t} / \mathrm{or} / \mathrm{d} /$ ? A quick look at the Subtlexus corpus (Brysbaert and New 2009) shows that this is not a trivial problem: of the 462,136 tokens which contain a surface flap, only $31.7 \%$ are in words that alternate $(9.3 \%$ with /d/ and $22.3 \%$ with $/ \mathrm{t} /)$. The other $68.3 \%$ are ambiguous non-alternating flaps, meaning that they could equally reasonably be mapped to either $/ \mathrm{t} /$ or $/ \mathrm{d} / .^{2}$

Here I explore two prominent theories of how learners treat cases of morphophonological ambiguity: Lexicon Optimization (Prince and Smolensky 2004) and the Free Ride Principle (McCarthy 2005).

Lexicon Optimization (hereafter LO) states that in cases of ambiguity when there is no contrary evidence, the underlying phonological representations are stored as the same as the surface form (Prince and Smolensky 2004). For example, LO predicts that an English speaker hearing a new word containing a word-medial flap (such as Ryder) will store the underlying representation as a flap, even though if alternations were discovered it could be the case that the true underlying representation is a $/ \mathrm{t} /$ or $/ \mathrm{d} /$.

The Free Ride Principle (hereafter FRP) states that "learners who have discovered the /A/ $\rightarrow$ [B] unfaithful map from alternations will attempt to generalize it, projecting /A/ inputs for all surface [B]s, whether they alternate or not" (McCarthy 2005). The FRP predicts that an English speaker who hears a non-alternating word with a medial flap (as in Ryder) should map that flap onto either $/ \mathrm{t} / \mathrm{or} / \mathrm{d} /$, following the independently-motivated /t/ $\rightarrow[\mathrm{r}]$ or /d/ $\rightarrow[\mathrm{r}]$ rules in English. The FRP makes no predictions about which of the two phonemes the non-alternating flap should map on to, though several potential factors are discussed in the General Discussion (Section 4).

To investigate which of the above strategies learners use, I analyze the case of flapping in Standard American English. In Standard American English, /t/ and /d/ neutralize to [r] intervocalically, after stressed vowels and before unstressed vowels. ${ }^{3}$ The majority of words which contain a surface flap, however, are words in which the surface flap does not alternate with either $/ \mathrm{t} /$ or $/ \mathrm{d} /$ (as in Ryder). The motivation for this experiment is the commonly observed fact that speakers of Canadian Raising ${ }^{4}$ dialects raise the /aI/ in spider [spsir $x$ ]: this suggests that the representation of this word is /spart $x /$, with the voiceless / $/$ / causing /aI/ to raise to $[\Lambda \mathrm{I}]$, then neutralizes to $[\mathrm{r}]$. If this is the case, this provides evidence for the Free Ride Principle over

\footnotetext{
* This work was supported by a College of Arts and Letters Undergraduate Research Initiative grant at Michigan State University and a Baggett Fellowship at the University of Maryland. I would like to thank Karthik Durvasula, Bill Idsardi, Elan Dresher, Margaret Kandel, Maxime Papillon, Michigan State University's Phonology Group, and the audiences at GLEEFUL 2017 and AMP 2017. All errors are my own.

1 At least four distinct taps and flaps have been distinguished (Derrick and Gick 2011). Here I use "flap" as a cover term to include all these.

2 The author manually coded each unique word containing a surface flap in this corpus (determined by extracting words which, according to the CMU dictionary, contain a T or D after a stressed vowel and before an unstressed vowel) for whether there exist morphological alternations which show that the flap is underlyingly a / $\mathrm{t} / \mathrm{or} / \mathrm{d} /$.

3 This is a slight simplification but suitable for present purposes.

4 Canadian Raising is a process in which the nucleus of /aI/ raises to [AI] before voiceless consonants (Joos 1975).
} 
Lexical Optimization, as LO would predict that the non-alternating flap should be represented underlyingly as a voiced $/ \mathrm{r} /$, which would not cause /ai/ raising. The way that language users treat non-alternating flaps in words like "spider" or "Ryder" can consequently weigh upon the debate between the Free Ride Principle and Lexicon Optimization theories.

If language users treat non-alternating flaps similarly to either the flap allophone of /t/ or the flap allophone of $/ \mathrm{d} /$, this provides support for the Free Ride Principle. If, on the other hand, speakers treat the non-alternating flap as different than the flap allophone of $/ \mathrm{t} /$ and than the flap allophone of $/ \mathrm{d} /$, this is evidence for Lexicon Optimization.

1.1 The present experiments This study analyzes the characteristics of the vowel preceding flaps in three environments: before flapped /t/ (writer), before flapped /d/ (rider), and before an ambiguous nonalternating flap (Ryder). Note that in all the words analyzed, both the non-alternating flaps and the $/ \mathrm{t} /$ and /d/ phonemes are produced as flaps; words in which these phonemes are realized as other allophones (such as $\left[\begin{array}{lll}t^{\mathrm{h}} & \mathrm{d} & \left.\mathrm{t}^{\mathrm{t}}\right]\end{array}\right]$ ) are excluded. This controls for any acoustic differences that might be found as a result of articulatory differences. The duration of the vowel preceding the flap in the three environments is analyzed in a production experiment (Experiment 1) and in a corpus of mostly spontaneous speech (Experiment 2).

Previous studies have found that flapped / $/$ / and flapped /d/ in Standard American English are incompletely neutralized on the surface and are distinguished by the duration of the preceding vowel (Braver 2011). ${ }^{5}$ Vowel durations are longer before voiced consonants, such that if language users treat the nonalternating flap as an allophone of $/ \mathrm{d} /$, the duration of the preceding vowel will be longer than the vowel before a flapped / $t /$ (and vice versa). If language users treat the non-alternating flap as distinct from both flapped / $\mathrm{t} /$ and flapped /d/, however, then one would expect differences in preceding vowel durations between all three conditions.

Experiment 1 additionally analyzes the F1 trajectory of a preceding /ai/ vowel. The predictions based on F1 trajectories are parallel to those regarding vowel durations: If language users treat this non-alternating flap as an allophone of /d/, raising is not expected, and the F1 of /al/ in these two environments should be similar to each other and significantly different than before flapped / $t /$. If language users treat the non-alternating flap as an allophone of $/ \mathrm{t} /$, raising is expected and the F1 of /aI/ in these two environments should be similar to each other and significantly different than before flapped /d/. If, however, speakers treat the non-alternating flap as something entirely separate from /t/ and /d/, as predicted by LO, we would expect the F1 trajectories in these three environments to all be significantly different from one another. ${ }^{6}$

\section{Experiment 1: Production Experiment}

The production experiment is designed to use Canadian Raising and vowel durations to investigate whether language users are treating the non-alternating flap similarly to the flap allophones of $/ \mathrm{t} / \mathrm{or} / \mathrm{d} /$, or as something entirely different. This was investigated by comparing acoustic data from similar words which contain /aI/ followed by flapped /t/, flapped /d/, and the ambiguous flap. It is expected that, if language users are treating the non-alternating ambiguous flap as a voiceless consonant, i.e. as /t/, they will raise /ai/ when it occurs before this segment (as in Ryder) to the same extent that they raise /aI/ before other surface flaps which are clearly allophones of /t/ (as in writer). Similarly, the vowel durations before flapped $/ \mathrm{t} /$ and non-alternating flap will be similar to each other and significantly different from durations before flapped $/ \mathrm{d} /$. However, if language users are treating the ambiguous flap as voiced, i.e. as /d/, they will not raise the /aI/ which comes before it, and the height of /aI/ will be similar to before a flapped /d/ (as in rider). Vowel durations would be similar before flapped /d/ and non-alternating flaps, and different from before flapped /t/.

\footnotetext{
5 Note, however, that Braver (2011) study categorizes words as containing /t/ or /d/ based on whether they are spelt with "t" or "d", respectively, and not on morphophonemic alternations, as the current study does.

6 A strict reading of the Canadian Raising rule - raise /ai/ before voiceless stops - actually predicts that /d/ and /r/ should pattern similarly, since neither are voiceless stops. However, previous results (Dickerson 2017) have shown that speakers do raise /aI/ before non-alternating flaps higher than before voiced stops (cider is higher than cyber) but not as high as before voiceless stops (cider is lower than cycle). This suggests that there may be another process besides Canadian Raising causing the differences observed here. However, what this other process may be is irrelevant to the current discussion; what matters is that there are reliable differences in F1 in these three environments, which does not follow if speakers treat the non-alternating flaps as allophones of $/ \mathrm{t} / \mathrm{or} / \mathrm{d} /$.
} 
If non-alternating flaps are treated differently than both flapped $/ \mathrm{t} /$ and flapped $/ \mathrm{d} /$, then the preceding vowel duration and F1 will be different in all three conditions.

2.1 Methods Data were collected in a sound attenuated room using a Samsung Meteor microphone (Frequency response: $20 \mathrm{~Hz}-20 \mathrm{KHz}$ ). An online survey platform Jotform (Jotform Inc. 2017) was used to present participants with a word list and demographic questions.

2.1.1 Participants Participants $(\mathrm{n}=14)$ were undergraduates in linguistics courses at Michigan State University. Participants were offered extra credit for participating. All of the participants were born and raised in Michigan during at least the first 8 years of life, did not report any language or hearing problems, and learned English exclusively during at least the first 8 years of life. All the participants self-identify as female and as white/caucasian.

2.1.2 Materials The word list consisted of 108 items: 18 test items and 90 fillers. Words were randomized into one list. Six sets of test tokens were formed such that each set contained 3 words that begin with the same initial segment, followed by /ar/, followed by a segment which surfaces as [r]: either /t/, $/ \mathrm{d} /$, or a non-alternating ambiguous flap, as shown in Table $1 .^{7}$ It was important to control for the segment preceding /aI/, as it has been shown that the preceding segment affects the height of the nucleus (K. Hall 2005). Nonce words were used to fill lexical gaps, where real English words either do not exist or are very infrequent. In order to obscure the purpose of the nonce words, about half of the fillers $(n=42)$ were also nonce words. Participants were asked to produce each word twice in a row to mitigate the potential for participants producing the first token of each pair disfluently, particularly for the nonce words.

Table 1: Experiment 1 test words.

\begin{tabular}{|c|c|c|c|}
\hline & $\begin{array}{c}/ \mathrm{t} / \mathrm{H} \\
\text { bimorphemic }\end{array}$ & $\begin{array}{c}/ \mathrm{d} / \\
\text { bimorphemic }\end{array}$ & $\begin{array}{c}\text { non-alt }[\mathrm{r}] \\
\text { monomorphemic }\end{array}$ \\
\hline $\mathrm{r}$ & riding & writer & Ryder \\
\hline $\mathrm{s}$ & sided & sighting & cider \\
\hline br & rider & brighter & bridle \\
\hline $\mathrm{f}$ & feiding & fighting & Fido \\
\hline $\mathrm{t}$ & tiding & tighter & taiderr \\
\hline h & hider & fighter & Heidi \\
\hline
\end{tabular}

Figure 1: Screenshot of Jotform presentation. Participants were asked to repeat each unique word twice.

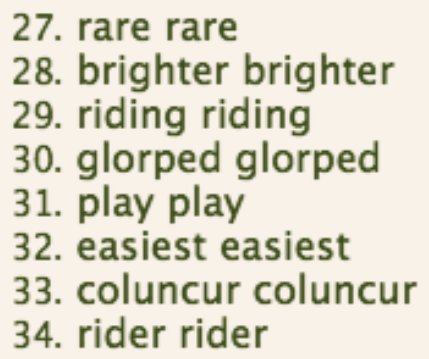

\footnotetext{
7 All of the non-alternating words used are spelt with a "d". This is because there are no common non-alternating monomorphemic English words which contain /aI/ followed by [r] which are spelt with "t". (Two potential words, vital and title alternate with vitality and titular, both clearly produced with allophones of $/ \mathrm{t} /$.)
} 
2.2 Data analysis The audio files were force-aligned using the Penn Phonetics Lab Forced Aligner (Yuan and Liberman 2008). After alignment, the test tokens were extracted and the /as/ vowel boundaries manually verified or corrected in Praat (Boersma and Weenink 2017). Tokens that were pronounced disfluently were removed before the analysis; these include tokens that were mispronounced due to speaker error (e.g. pronouncing "bridle" as "brittle") or due to hypercorrection. ${ }^{8}$ In total, $19 \%$ of the tokens were excluded. A Praat script extracted the F1 and F2 for all remaining tokens of /aI/ at 20 evenly spaced times points in each vowel, as well as the duration of each vowel.

\subsection{Results}

2.3.1 F1 A within-subjects ANOVA shows that the following segment has a significant effect on the F1 of the preceding vowel; $F(2,26)=60.4, p<0.0001$. Planned comparison Welch t-tests show that the $F 1$ of /aI/ is significantly higher before flapped $/ \mathrm{d} /(\mathrm{M}=699.4 \mathrm{~Hz})$ than before non-alternating flaps $(\mathrm{M}=649.3$ $\mathrm{Hz}) ; \mathrm{t}(25.9)=1.9, \mathrm{p}=0.03]$ and higher before non-alternating flaps $(\mathrm{M}=649.3 \mathrm{~Hz})$ than before $/ \mathrm{t} / \mathrm{M}=$ $617.7 \mathrm{~Hz}) ;[\mathrm{t}(25.6)=2.0, \mathrm{p}=0.03]$. Figure 2 plots this data. artifact.

Figure 3 shows that there is no bimodality in this data, suggesting that this is not simply an averaging

Figure 2: Change of F1 values through the /aI/ vowel, separated by the following segment.

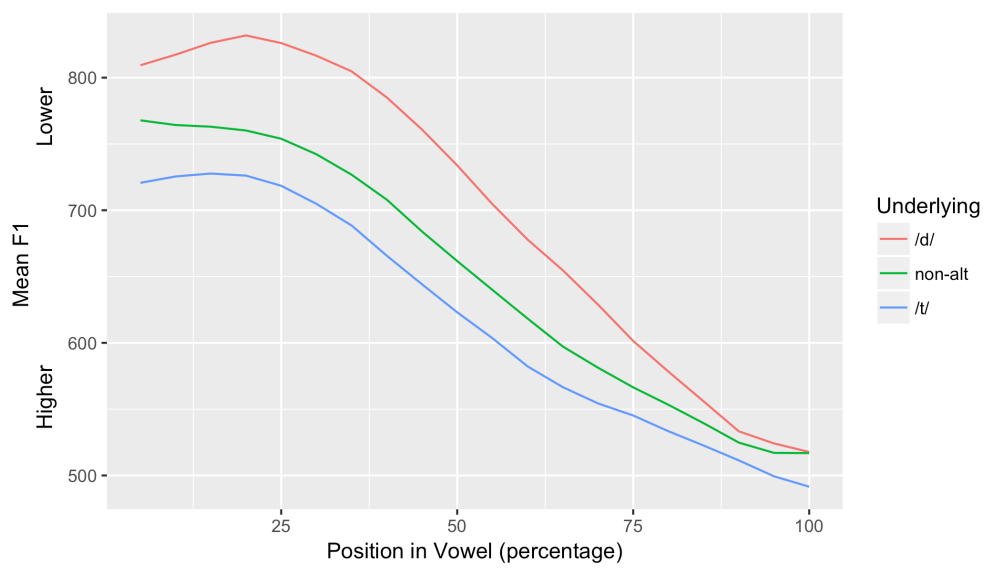

8 Because of the oddness of the task, a word list with many nonce words, participants oftentimes hypercorrected, producing word-medial " $\mathrm{t}$ " as $\left[\mathrm{t}^{\mathrm{h}}\right]$. Because the relevant comparison is between vowels which precede flaps and because of the articulatory differences between a flap and $\left[\mathrm{t}^{\mathrm{h}}\right]$, words which were produced without flaps were excluded. 
Figure 3: Violin plots showing the distribution of $\mathrm{F} 1$ values at three time points: $25 \%, 50 \%$, and $75 \%$ through the vowel /aI/. Each data-point is an individual token: the horizontal jitter is not meaningful and is only used visualization purposes. The violin plots show no bi-modality, suggesting that the results are not simply an averaging effect.

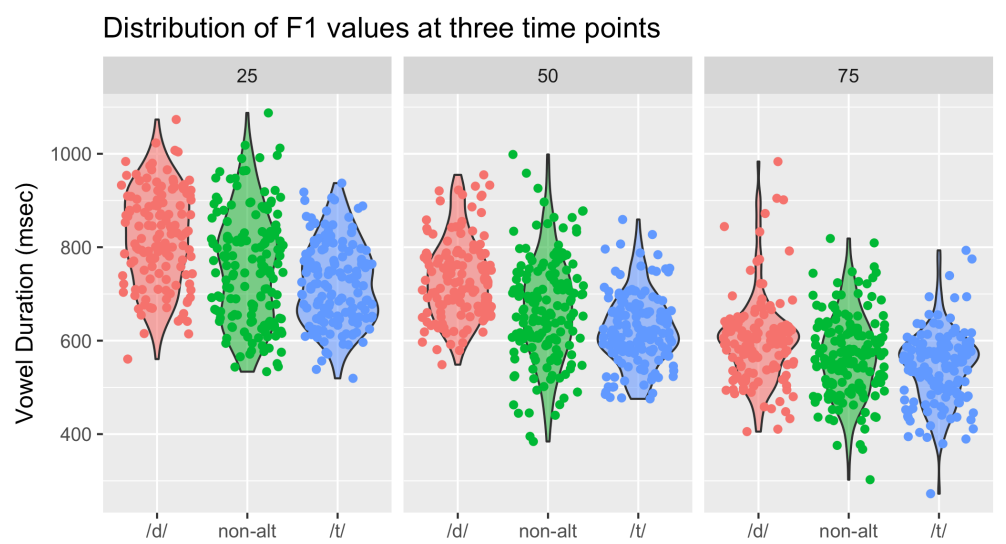

2.3.2 Vowel duration A within-subjects ANOVA shows that the following environment has a significant effect on the vowel duration: $\mathrm{F}(2,26)=15.5, \mathrm{p}<0.0001$. Planned comparison Welch t-tests show that the vowel durations of /aI/ before $/ \mathrm{d} /(\mathrm{M}=176.3 \mathrm{~ms})$ are not significantly longer than those before non-alternating flaps $(\mathrm{M}=162.3 \mathrm{~ms}) ; \mathrm{t}(25.1)=0.7, \mathrm{p}=0.2]$ though they are marginally significantly longer before nonalternating flaps $(\mathrm{M}=162.3 \mathrm{~ms})$ than before $/ \mathrm{t} /(\mathrm{M}=149.7) ; \mathrm{t}(19.9)=1.5, \mathrm{p}=0.07]$. Figure 4 plots this data.

Figure 4: Box plots showing the distribution of vowel duration values. Each data-point is an individual token: the horizontal jitter is not meaningful and is only used visualization purposes. The box plots show no bi-modality, suggesting that the results are not simply an averaging effect.

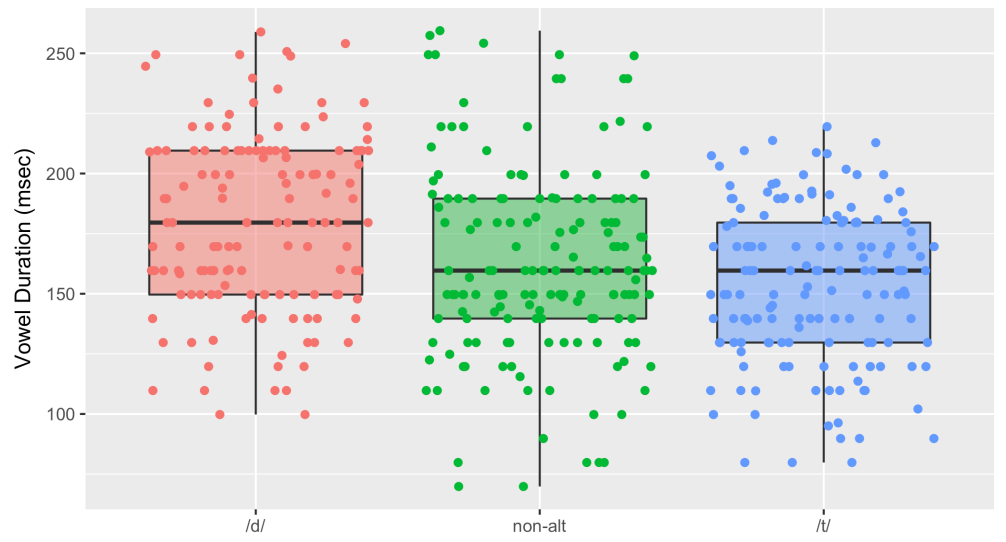

2.4 Discussion In Experiment 1, the vowel duration and the F1 trajectories of /aI/ preceding surface flaps were analyzed. The predictions were that if the non-alternating flap is treated by language users more like an allophone of $/ \mathrm{t} /$, the preceding /aI/ would have vowel durations and $\mathrm{F} 1$ trajectories comparable to before flapped /t/ (shorter duration and raised nucleus) versus before flapped /d/. Similarly, if language users treat the non-alternating flap as an allophone of /d/, the preceding /aI/ would have vowel durations and F1 trajectories 
comparable to before flapped /d/ (longer duration and un-raised nucleus) versus before flapped /t/. Both of these results would be compatible with the Free Ride Principle. If, however, the non-alternating flap behaves differently than both flapped /t/ and flapped /d/, then preceding vowel durations and F1 trajectories can be different in all three environments: before flapped $/ \mathrm{t} /$, before flapped $/ \mathrm{d} /$, and before a non-alternating flap. This is possible under Lexicon Optimization.

The findings show that, for both F1 and vowel durations, the vowel is treated differently in all three environments (though the distinction is less clear for vowel durations). The F1 of /aI/ before a non-alternating flap is significantly different than before flapped / $/$ / and flapped /d/ (and marginally so for vowel durations); providing evidence against the FRP. The results suggest that language users are not simply mapping nonalternating flap onto /t/ or onto /d/. Additionally, no bi-modality is present in the data, which suggests that this is not simply an averaging effect, i.e., speakers are not treating the non-alternating flap as derived from /t/ half the time and from /d/ the other half of the time. The results present a challenge to the Free Ride Principle and support for Lexicon Optimization.

The actual differences between the three environments, though they are statistically significantly different from each other, are very small and thus may not be meaningfully different. That is, they may not be differences that are attended to by language users during speech perception or by language learners during acquisition. I leave ascertaining this for future work.

As noted above, nearly $20 \%$ of test words had to be excluded in this task. This is likely due to the awkwardness of word lists, as many participants pronounced everyday words incorrectly, and the use of nonce words, which caused many disfluencies. Though this task provided valuable controlled data, reading speech is known to be different than free speech and more naturalistic data could provide valuable insights. I conducted a follow-up experiment analyzing acoustic data from a corpus of spoken speech using nearly the same predictions and design as the production task.

\section{Experiment 2: Corpus Analysis}

Because of the somewhat unnatural nature of any production experiment described above, a speech corpus was used to analyze more naturalistic data. I analyzed the durations of vowels preceding surface flaps in the same three environments as Experiment 1: before a flapped /d/, before a flapped $/ \mathrm{t} /$, and before an ambiguous non-alternating flap.

The question and predictions are the same as those in Study1. Analyzing preceding vowel durations can shed light on whether speakers are treating the ambiguous non-alternating flaps more like voiced or voiceless stops (vowel durations should be longer if the following consonant is voiced than if it is voiceless, and vice versa) or as something different entirely.

3.1 Methods The IHELP corpus (Wagner et al. 2016) contains sociolinguistic interviews with native English speakers who grew up in Lansing, Michigan. The participants completed three tasks besides the interview: acceptability judgments, semantic differentials, and a word list. The data reported here are from 26 speakers, all white females, to mirror the demographic makeup of the participants of Experiment 1. The data analyzed includes speech from the interview portion as well as the three other tasks.

The speech in the corpus for the speakers analyzed does not include any words which contain the vowel /aI/ preceding a flap. Consequently, only vowel duration was analyzed; vowel formants were not examined for this dataset.

The interviews were transcribed and force-aligned, then the duration of each segment was extracted using a Praat script (Boersma and Weenink 2017). All bisyllabic words containing a word-medial surface flap (as determined by the CMU Pronouncing Dictionary (Weide 1994)) were extracted and manually coded for whether the word has morphophonemic alternations and if the segment underlying the flap was a $/ \mathrm{t} / \mathrm{a} / \mathrm{d} /$, or unknown. Unfortunately, only two vowels, /i/ and /I/, occurred in all three environments (before flapped $/ \mathrm{d} /$, before flapped $/ \mathrm{t} /$, and before non-alternating flap) and there were not enough tokens of these words to merit analysis; therefore, analysis by individual vowel was impossible. Instead, the distinction considered is between tense (i e a o u) and lax ( $\varepsilon æ \supset$ ) vowels; diphthongs are excluded.

The corpus, subsetted to the 26 speakers, contains 615,717 transcribed words. Of these, 603 tokens ( $<$ $0.1 \%$ of total words), consisting of 61 unique words, are bisyllabic words which contain a surface flap. Tokens which were more than two standard deviations from the category mean were excluded from the analysis; this 
resulted in the exclusion of 25 tokens ( $4 \%$ of the total).

3.2 Results Figure 5 shows the vowel durations of tense and lax vowels in the three environments, but the sparseness of data, as shown in Table 2, makes it unenlightening to compare any but the tense vowels followed by non-alternating flaps to those before flapped /t/. A directional Welch $\mathrm{t}$-test shows that the tense vowels before non-alternating flaps are significantly longer $(\mathrm{M}=138.4 \mathrm{~ms})$ than tense vowels before flaps derived from $/ \mathrm{t} /(\mathrm{M}=93.9 \mathrm{~ms}) ;[\mathrm{t}(33.4)=5.3, \mathrm{p}<0.0001]$.

Figure 5: Plot showing the durations (in milliseconds) of vowels preceding surface flaps in the IHELP corpus. Box plots show the mean and quartiles of the distribution. Each data-point represents one token (the horizontal jitter is not meaningful and is only used visualization purposes); note the unequal distribution of tokens across conditions.

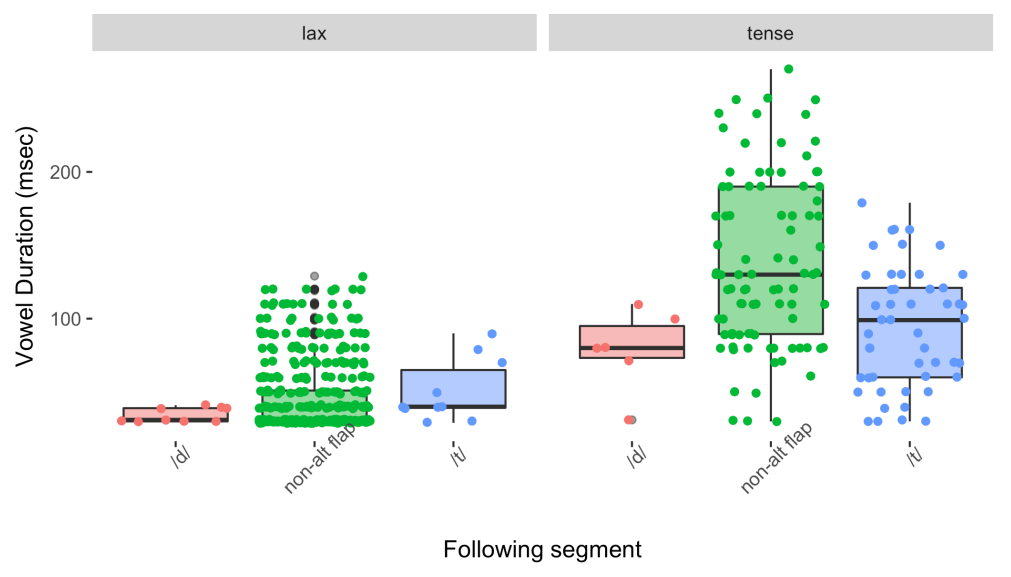

Table 2: Number of tokens in subsetted corpus containing surface flaps, split up by the flap's underlying phoneme and the tense/laxness of the preceding vowel.

\begin{tabular}{l|cc} 
& lax & tense \\
\hline$/ \mathrm{d} /$ & 9 & 6 \\
non-alt $[\mathrm{r}]$ & 438 & 91 \\
$/ \mathrm{t} /$ & 10 & 49
\end{tabular}

3.3 Discussion While corpus data is in general a valuable naturalistic complement to elicited lab data, there exist a few concerns related to the use of this corpus in the present study, so the following discussion should be taken with a grain of salt. The contents of the interviews were not controlled, leading to unequal distributions of tokens across environments. This makes interpreting and generalizing from the data difficult. Additionally, I had access only to the measurements of each segment in this corpus, and not the original audio files, therefore, I was unable to exclude mispronounced or hyper-corrected tokens from the analysis. Finally, I was unable to separately analyze the different styles of speech (spontaneous vs. semantic differential vs. word list).

Those caveats aside, two major findings can be identified in the present results. First, the tense vowels appearing before non-alternating flaps are longer than those before flapped / $t$. This is consistent with the non-alternating flaps being voiced; however, there is too little data regarding vowel lengths before flapped /d/ to be sure. Second, the token frequencies in this speech corpus mirror those of the Subtlexus corpus: there are many more ambiguous flap tokens than clearly /d/- or /t/-derived flap tokens. 


\section{General Discussion}

4.1 Summary This study examined the relationship between $/ \mathrm{t} / \mathrm{d} / \mathrm{d} /$, and non-alternating flaps in Michigan English by analyzing the durations and F1 trajectories of the preceding vowel in data from a production experiment (Experiment 1) and a corpus of spontaneous speech (Experiment 2). In both Experiment 1 and Experiment 2, significant differences were found for vowels appearing in the three environments. These results from production data provide evidence for the theory of Lexicon Optimization: language users are treating the non-alternating flap as something different than both flapped /t/ and flapped /d/. If language users made use of the Free Ride Principle in their representation of non-alternating flaps, it would be expected that such flaps should behave either like flapped /t/ or like flapped /d/.

4.2 Future work However, it remains to be seen whether these differences in production are perceived as meaningful by language users. As noted in the results section of both Experiment 1 and Experiment 2, the actual differences between the three conditions are very small, even though they are statistically significant. A perception task is being designed to investigate whether listeners pay attention to these small differences in vowel lengths and F1 trajectories and whether these cues are attended to by language learners.

In addition, other experiments are currently being developed in order to provide a more nuanced understanding of the current results, as they do not rely on notoriously messy acoustic data to draw conclusions about abstract underlying representations. A morpheme relatedness test (similar to that reported in (K. C. Hall et al. 2016)) and an EEG priming task are being designed to investigate whether language users treat, for example, Ryder and writer as being more similar to each other than to rider, vice versa, or if language users treat all three of these categories as similarly different. An artificial language learning paradigm will investigate biases in learning: when a grammar contains a segment which does not behave as a phoneme and alternates with two phonemes equally frequently, but also appears in non-alternating words, do language users treat this segment as an independent phoneme (as predicted by LO) or map it onto one of the two phonemes it appears with (as predicted by FRP)?

\subsection{Implications}

4.3.1 The psychological reality of orthography Many authors make the assumption that words spelt with a " $t$ " contain a /t/ phoneme and those spelt with a "d" contain a /d/ phoneme, relying on spelling (which, presumably, first language learners do not have immediate access to) rather than on morphophonological alternations (which are more likely directly accessible to first language learners).

As one example, a recent paper (Warner and Tucker 2017) categorizes turtle and battle as containing word-medial /t/, and hurdle and cradle as containing word-medial/d/, even though these flaps never alternate with $/ \mathrm{t} /$ or $/ \mathrm{d} / .^{9}$

However, as shown by this study and many others, it is dangerous to assume that spelling reflects the psychological reality of language users' underlying representations. Nevins and Vaux (2008) present evidence that spelling can influence phonological representations when there is a lack of other types of evidence, but they also cite many examples of underlying representations conflicting with the spelling: "enchila[ $\left[\mathrm{t}^{\mathrm{h}}\right] \mathrm{a}$ ", "che[ $\left.\mathrm{t}^{\mathrm{h}}\right]$ ar cheese", and "somebo[ $\left.\mathrm{t}^{\mathrm{h}}\right] \mathrm{y}$ " are all pronounced with aspirated $\left[\mathrm{t}^{\mathrm{h}}\right]$ by at least some speakers. ${ }^{10}$ Conventional spelling is not a reliable indicator of underlying representations, though children's non-traditional spelling often provides valuable insights into their representations. As of January 2018, searching "spiter" on YouTube yields 7,350 results, including videos and playlists titled "Spiter man", "killer spiter", "killed giant spiter", and "SPITER-MAN vs HULK", among others. Searching "spiter" on Google yields 253 thousand results. Additionally, children will often spell words beginning with /s/+stop with the voiced counterpart of the stop consonant, e.g. "sbin", "sdop", and "sgy" (Hannam, Fraser, and Byrne 2007). These many examples show that we cannot rely on traditional spellings to indicate what the language users' underlying representations actually are.

\footnotetext{
9 This study was chosen as an example because of the similar design to the present study (both analyze the formants of the vowel preceding the flap in production data) and because in their analysis, they did not find a difference between words with what they categorize as /t/ vs /d/ and so collapsed these two groups. It may be illuminating to re-investigate their data with a three-level factor of underlying segment: /t/, /d/, and non-alternating flap.

${ }^{10}$ Similarly, the current author's wife produces "rea[ $\left.\mathrm{t}^{\mathrm{h}}\right] \mathrm{y}$ " in formal situations.
} 
Until we know more about what language users do when confronted with non-alternating words containing segments which do not otherwise behave like phonemes in their language, care should be taken in categorizing these words by spelling alone. There is no more reason to a priori assume that, for example, hurdle contains a /d/ than there is to assume that writer contains a /w/.

4.3.2 Revisiting the Free Ride Principle The results presented here provide support for Lexicon Optimization, since in production the three segments $(/ \mathrm{t} /, / \mathrm{d} /$, and non-alternating flap) behave differently. However, it remains to be seen whether these small differences in production translate into perceived differences by listeners and learners. Therefore, some remaining questions about the Free Ride Principle will be addressed here. If language users do use the Free Ride Principle, the question becomes how language users decide which of the two phonemes (/t/ or /d/) language users default to, though that was not the objective of this study. Two potential factors are word frequency and orthography.

Frequency could have an effect: If, for example, $80 \%$ of the surface flaps alternate with /t/, $10 \%$ alternate with $/ \mathrm{d} /$, and $10 \%$ are non-alternating, it would make sense for language users to map the non-alternating $[\mathrm{r}] \mathrm{s}$ onto /t/ via the Free Ride Principle and mark the $[r] \sim / d /$ alternations as simply exceptions. ${ }^{11}$ However, both the Subtlexus corpus of movie subtitles and the IHELP corpus of spontaneous speech show that the majority of surface flap tokens occur in non-alternating contexts. In this case, it becomes difficult to form coherent predictions about which of $/ \mathrm{t} /$ or $/ \mathrm{d} /$ the language learner maps the non-alternating flap onto.

The Perceptual Uncertainty Hypothesis (Daland, Oh, and Kim 2015) states that orthography is an important influence if perceptual cues underconstrain possible loanword adaptations. This hypothesis could be extended to L1 word learning: if perceptual cues (i.e. phonetic cues and morphophonological alternations) underconstrain possible underlying representations, language users may rely on orthography to determine their underlying representation. In this case, for example, Ryder might be adopted with a /d/ in the underlying representation because of the "d" in spelling. However, there do exist counterexamples to this: "enchila[ $\left.\mathrm{t}^{\mathrm{h}}\right] \mathrm{a}$ ", etc. (Nevins and Vaux 2008). It remains to be seen whether and to what extent orthography affects phonology, particularly when, as in the majority of cases, language learners hear the word before learning the spelling of it.

4.3.3 Implications of Lexicon Optimization This article presents results that show that the preceding vowels in the three environments (before flapped /t/, before flapped /d/, and before a non-alternating flap) behave significantly differently from each other, providing evidence for Lexicon Optimization over the Free Ride Principle. These results are somewhat surprising. If the underlying representation of non-alternating flaps is simply a flap rather than $/ \mathrm{t} / \mathrm{or} / \mathrm{d} /$, one might expect that these flaps have the same privileged status as other phonemes. However, they do not behave as other phonemes do in American English: they are completely predictable based on their surrounding environments (i.e. they always occur intervocalically) and do not discriminate words.

\section{References}

Boersma, Paul and David Weenink (2017). Praat: doing phonetics by computer [Computer program]. Version 6.0.36. URL: http: //www.praat.org/.

Braver, Aaron (2011). "Incomplete Neutralization in American English Flapping: A Production Study". In: Proceedings of the 34th Annual Penn Linguistics Colloquium 17.1, pp. 31-40. ISSN: 15249549.

Brysbaert, M and B New (2009). "Moving beyond Kucera and Francis: A Critical Evaluation of Current Word Frequency Norms and the Introduction of a New and Improved Word Frequency Measure for American English". In: Behavior Research Methods.

Daland, Robert, Mira Oh, and Syejeong Kim (2015). "When in doubt, read the instructions: Orthographic effects in loanword adaptation". In: Lingua 159, pp. 70-92. ISSN: 00243841. DOI: $10.1016 / \mathrm{j}$. lingua.2015.03.002. URL: http://dx.doi.org/10.1016/j.lingua.2015.03.002.

Derrick, Donald and Bryan Gick (2011). "Individual variation in English flaps and taps: A case of categorical phonetics". In: The Canadian Journal of Linguistics / La revue canadienne de linguistique.

Dickerson, Bethany (2017). Canadian Raising in Michigan English.

${ }^{11}$ Special thanks to Donca Steriade for bringing this to my attention. Bill Idsardi makes a similar point regarding neutralization in Korean (Idsardi 2006). 
Hall, Kathleen (2005). "Defining phonological rules over lexical neighbourhoods: Evidence from Canadian Raising". In: Proceedings of the 24th West Coast Conference on Formal Linguistics.

Hall, Kathleen Currie et al. (2016). "Measuring perceived morphological relatedness". In: The Canadian Journal of Linguistics/La revue canadienne de linguistique.

Hannam, Rachel, Helen Fraser, and Brian Byrne (2007). “The sbelling of sdops: Preliterate children's spelling of stops after /s/". In: Reading and Writing 20, pp. 399-412.

Idsardi, William (2006). "A Bayesian Approach to Loanword Adaptations”. In: LSA. January.

Joos, Martin (1975). "A phonological dilemma in Canadian English". In: ed. by J.K. Chambers. Methuen.

Jotform Inc. (2017). Jotform. URL: http: / / www. jot form. us.

McCarthy, John (2005). "Taking a Free Ride in Morphophonemic Learning". In: Catalan Journal of Linguistics 4.January, pp. 19-55. DOI: 10.2307/3453757.

Nevins, Andrew and Bert Vaux (2008). "Underlying Representations that do not Minimize Grammatical Violations". In: Studies in Generative Grammar : Freedom of Analysis?, pp. 35-61. URL: http : / / discovery.ucl.ac.uk/68236/.

Prince, Alan and Paul Smolensky (2004). "Optimality: constraint interaction in generative grammar". In:

Wagner, Suzanne Evans et al. (2016). "Reversal and re-organization of the Northern Cities Shift in Michigan". In: University of Pennsylvania Working Papers in Linguistics: Selected Papers from NWAV 44.

Warner and Tucker (2017). "An effect of flaps on the fourth formant in English". In: Journal of the International Phonetic Association.

Weide, R.L. (1994). CMU Pronouncing Dictionary. URL: http: / / www . speech. cs . cmu . edu/cgibin/cmudict.

Yuan, Jiahong and Mark Liberman (2008). "Speaker identification on the SCOTUS corpus". In: Proceedings of Acoustics. 